\title{
Bioévaluation du potentiel d'eutrophisation du phosphore des lixiviats de boues de forage pétrolier
}

\author{
J.E. Djomo1 \\ A. Dauta ${ }^{1}$ \\ F. Moreau ${ }^{2}$
}

Mots clés : bioévaluation, potentiel d'eutrophisation, Selenastrum capricornutum, phosphore, lixiviats, boues de forage pétrolier.

Des bioessais avec l'algue Selenastrum capricornutum Printz ont été intégré en 1991 à un programme d'étude élaboré par le département Environnement de la société Elf Aquitaine Production de Boussens ; ils étaient destinés à l'évaluation en milieu d'eau douce, du potentiel eutrophisant du phosphore des lixiviats de trois boues de forage pétrolier. Le paramètre suivi est la biomasse maximale produite.

Les résultats obtenus sont discutés en vue d'une évaluation et d'une correction éventuelle du potentiel d'eutrophisation des lixiviats des boues de forage. Ceci permet d'optimiser la stratégie de gestion de ces types de déblais. Les lixiviats de ceux-ci ont un potentiel eutrophisant relativement faible.

Bioassessment of the eutrophication potential in relation to phosphorus leaching from petroleum drilling muds

Keywords : bioassessment, eutrophication potential, Selenastrum capricornutum, phosphorus, leaching, petroleum drilling muds.

Bioassay using Selenastrum capricornutum Printz, as test-organism was adopted in an environmental research programme conceived in 1991 by the société Elf Aquitaine Production of Boussens. The aim of this study was to evaluate, the eutrophication potential in relation to phosphorus leaching from petroleum drilling muds. The parameter measured was the yield.

The results are discussed in order to optimize the appropriate strategy for those muds. They showed that the leaching had a relatively low eutrophication potential.

\section{Introduction}

Le déversement de divers rejets industriels dans les milieux naturels contribue à les dégrader. Cette dégradation est souvent consécutive à l'accumulation dans les milieux récepteurs d'éléments minéraux et/ou organiques. Parmi ces éléments, le phosphore reste sans nul doute, l'élément essentiel qui est à l'origine de l'eutrophisation des plans d'eau (Saltman \& Heuer 1985, Cluis et al. 1989).

1. Laboratoire d'Hydrobiologie, URA 695 CNRS, Université Paul Sabatier, 118 route de Narbonne, 31062 Toulouse Cedex, France.

2. Laboratoire du Département Environnement, Société Elf Aquitaine Production, 31360 Boussens, France.
L'analyse chimique des divers polluants dans les eaux constitue le moyen d'investigation habituel. Celle-ci mesure en effet la pollution brute et non la pollution nette c'est-à-dire celle qui agit réellement sur les microorganismes. Dans le cas précis de l'eutrophisation, le phosphore contenu dans le milieu n'est que partiellement biodisponible.

L'utilisation des bioessais peut permettre de préciser l'importance des diverses fractions de phosphore (Lacaze 1987, Watanabe et al. 1988, Lacaze et al. 1989, Van Donk et al. 1989, Couture 1990).

Le principal but de ce travail est d'évaluer le potentiel d'eutrophisation des lixiviats de trois boues non solidifiées de forage pétrolier. L'organisme-test utilisé est l'algue Selenastrum capricornutum Printz de la famille des chlorophycées (Bourrelly 1966). Ces 
boues ont été étudiées à la Société Elf Aquitaine Production pour les aspects suivants :

- caractérisation physico-chimique ;

- solidification ;

- bactériologie ;

- tests d'écotoxicité et de germination.

A notre connaissance, aucune recherche spécifique de bioévaluation du potentiel eutrophisant du phosphore des boues étudiées n'a encore été effectuée.

\section{Matériel et méthodes}

\subsection{Mátériẹl}

Le matériel d'étude est constitué par un organisme-test et des échantillons d'eau (lixiviats).

L'organisme-test est une algue verte planctonique : Selenastrum capricornutum (Chlorophycées) (Bourrelly 1966). Cette algue est très utilisée pour les tests de fertilité des eaux polluées ; ses qualités ont été longuement évoquées par Lacaze et al. (1989), lors d'une étude menée sur la bioévaluation de la pollution des sédiments de la Seine. La souche utilisée a été fournie par la direction "Qualité des eaux » du CEMAGREF (Centre National du Machinisme Agricole du Génie Rural des Eaux et des Forêts) de Bordeaux.

Les lixiviats testés sont des lixiviats de trois boues non solidifiées de forage pétrolier. Ils ont été préparés conformément à la norme AFNOR (NFX 31-210) : mélange de 100 grammes de boue (préalablement séchée à l'étuve à $50^{\circ} \mathrm{C}$ et tamisée à $4 \mathrm{~mm}$ ) et de 1 litre d'eau déminéralisée ; agitation sur une table pendant 16 heures à la fréquence de 60 tours/minute puis décantation et filtration à $0,45 \mu \mathrm{m}$ (filtre en verre de type Whatman GF/G). Les caractéristiques physico-chimiques des lixiviats sont récapitulées sur le tableau 1.

\subsection{Paramètre expérimental mesuré}

Le paramètre choisi est la biomasse maximale produite. Le choix de ce paramètre tient au fait que la biomasse maximale produite est fiable pour des bioessais n'excédant pas 21 jours (Hanna 1985) ; ce qui est le cas de nos expérimentations.

\subsection{Méthodes}

\section{- Bioessais}

Les expériences ont été conduites dans des erlenmeyers en verre de borosilicate de $500 \mathrm{ml}$. Le milieu de culture (Tableau 2) est celui qui a été proposé par Dauta (1982) ; il est essentiellement minéral afin d'éviter la prolifération bactérienne. Les cultures sont réalisées en milieu artificiel non renouvellé (Batch-culture) avec un apport d'air (aération continue et agitation) de $0,5 \mathrm{l} / \mathrm{mn}$ de culture. Une aération continue permet de limiter l'appauvrissement du milieu en dioxyde de carbone. Une agitation quotidienne des cultures permet de maintenir les algues en suspension.

Les bioessais sont réalisés avec des lixiviats qui ont préalablement subi deux types de traitement : une filtration à $0,45 \mu \mathrm{m}$ (filtre de verre de type Whatman GF/C) et un autoclavage $\left(120^{\circ} \mathrm{C}\right.$ pendant $15 \mathrm{mn}$ ). Ces traitements tout en modifiant les

Tableau 1. Caractéristiques physico-chimiques des lixiviats. $\left(^{*}\right)$ phosphore total ; $\left({ }^{* *}\right)$ hydrocarbures totaux.

Table 1. Physical and chemical characteristics of leached materials. $\left({ }^{*}\right)$ total phosphorus ; $\left({ }^{* *}\right)$ total hydrocarbons.

\begin{tabular}{|c|c|c|c|c|c|c|c|c|c|c|c|}
\hline $\begin{array}{c}\text { Echantillons } \\
:\end{array}$ & PH & $\begin{array}{l}\text { Turb. } \\
\text { (ntu) }\end{array}$ & $\begin{array}{l}\text { M.E.S } \\
(\mathrm{mg} / \mathrm{l})\end{array}$ & $\begin{array}{l}\text { C.O.T } \\
(\mathrm{mg} / \mathrm{l})\end{array}$ & $\begin{array}{c}\mathrm{Ca} \\
(\mathrm{mg} / \mathrm{l})\end{array}$ & $\begin{array}{l}\mathrm{N}-\mathrm{NO} 3 \\
(\mathrm{mg} / \mathrm{l})\end{array}$ & $\begin{array}{l}\mathrm{N}-\mathrm{NH4} \\
(\mathrm{mg} / \mathrm{l})\end{array}$ & $\begin{array}{c}\mathbf{N}-\mathbf{K j} \\
(\mathrm{mg} / \mathrm{l}) \\
\end{array}$ & $\begin{array}{l}\text { P-PO4 } \\
(\mathrm{mg} / \mathrm{l}) \\
\end{array}$ & $\begin{array}{l}\text { P.T(") } \\
(\mathrm{mg} / 1)\end{array}$ & $\begin{array}{l}\text { H.T(**) } \\
(\mathrm{mg} / \mathrm{l})\end{array}$ \\
\hline Lixiviat 1 & 9,6 & 39 & 0,016 & 64.42 & 17 & 0,016 & 0,11 & 4,98 & 0,03 & 0.08 & 2,6 \\
\hline Lixiviat 2 & 7,7 & 6 & 0,002 & 176.7 & 625 & 0,097 & 0,08 & 3,64 & 0.01 & 0,018 & 2,2 \\
\hline Lixiviat 3 & 7,9 & 2 & 0,03 & 25,5 & 494 & 0,012 . & 0,11 & 2,9 & 0.01 & 0,027 & 1,4 \\
\hline
\end{tabular}


Tableau 2. Composition chimique du milieu de culture $(\mathrm{pH}=7 \pm 0,04)$.

Table 2. Chemical composition of culture medium $(\mathrm{pH}=7 \pm 0,04)$.

\begin{tabular}{|c|c|c|c|}
\hline Substances nutritives & & $\begin{array}{l}\text { Concentrations de } \\
\text { la Solution-mère }\end{array}$ & $\begin{array}{l}\text { Concentrations finales } \\
\text { de la solution d'essai }\end{array}$ \\
\hline & $\mathrm{MgSO} 4,7 \mathrm{H} 2 \mathrm{O}$ & $25 \mathrm{~g} / \mathrm{l}$ & $25 \mathrm{mg} / \mathrm{l}$ \\
\hline & $\mathrm{FeSO} 4,7 \mathrm{H} 2 \mathrm{O}$ & $1 \mathrm{~g} / \mathrm{l}$ & $1 \mathrm{mg} / \mathrm{l}$ \\
\hline & $\mathrm{CaCl} 2,2 \mathrm{H} 2 \mathrm{O}$ & $25 \mathrm{~g} / \mathrm{l}$ & $25 \mathrm{mg} / \mathrm{l}$ \\
\hline \multirow[t]{6}{*}{ Milieu de base } & NaEDTA, $2 \mathrm{H} 2 \mathrm{O}$ & $1 \mathrm{~g} / \mathrm{l}$ & $1 \mathrm{mg} / \mathrm{l}$ \\
\hline & $\mathrm{NaHCO} 3$ & $50 \mathrm{~g} / \mathrm{l}$ & $50 \mathrm{mg} / \mathrm{l}$ \\
\hline & $\mathrm{NaCO} 3$ & $5 \mathrm{~g} / 1$ & $5 \mathrm{mg} / \mathrm{l}$ \\
\hline & $\mathrm{ZnSO} 4,7 \mathrm{H} 2 \mathrm{O}$ & $20 \mathrm{mg} / \mathrm{l}$. & $20 \mu g / l$ \\
\hline & $\mathrm{CuCl} 2,4 \mathrm{H} 2 \mathrm{O}$ & $20 \mathrm{mg} / \mathrm{l}$ & $20 \mu g / l$ \\
\hline & $\mathrm{MnCl} 2,4 \mathrm{H} 2 \mathrm{O}$ & $400 \mathrm{mg} / \mathrm{l}$ & $400 \mu \mathrm{g} / 1$ \\
\hline \multirow[t]{4}{*}{ Oligo-éléments } & $\mathrm{CoCl} 2,6 \mathrm{H} 2 \mathrm{O}$ & $10 \mathrm{mg} / \mathrm{l}$ & $10 \mu \mathrm{g} / \mathrm{l}$ \\
\hline & H3BO3 & $1 \mathrm{mg} / \mathrm{l}$ & $1 \mu g / l$ \\
\hline & $\mathrm{NaNoO} 4$ & $35 \mathrm{mg} / 1$ & $35 \mu \mathrm{g} / \mathrm{l}$ \\
\hline & KNO3 & $200 \mathrm{~g} / \mathrm{l}$ & $200 \mathrm{mg} / \mathrm{l}$ \\
\hline Nutriments & $\mathrm{K} 2 \mathrm{HPO} 4$ & $25 \mathrm{~g} / 1$ & $25 \mathrm{mg} / \mathrm{l}$ \\
\hline
\end{tabular}

propriétés des lixiviats (élimination d'une partie du phosphore par filtration), présentent l'avantage de détruire les organismes indésirables (autoclavage). Certains organismes tels que les bactéries peuvent entrer en compétition avec l'algue-test pour l'assimilation des nutriments (Rhee 1972, Bayle 1980, Capblancq 1990).

L'inoculum utilisé provient d'une pré-culture carencée en phosphore durant 21 jours ; sa technique de préparation est analogue à celle de Hanna (1985) : tout en évitant l'épuisement éventuel des autres éléments nutritifs, le milieu de culture exempt de phosphore est renouvellé tous les 2 jours.

Les lixiviats en proportion croissante $(5,10,15$ ou $20 \%$ ) et $10^{4}$ cellules algales $/ \mathrm{ml}$ sont inoculés dans le milieu de culture comprenant tous les éléments à l'exception du phosphore. Parallèlement, une gamme-étalon a été réalisée avec le milieu de culture ne contenant que des cellules algales $\left(10^{4} / \mathrm{ml}\right)$ et des concentrations initiales croissantes $(1,10,25,50,100$, 200 et $300 \mu \mathrm{g} / \mathrm{l})$ de phosphore sous forme $\mathrm{K}_{2} \mathrm{H} \mathrm{PO}_{4}$.
Les conditions de cultures sont les suivantes :

- éclairement par une rampe constituée de tubes florescents blancs (type F36T8/CW) donnant une intensité lumineuse de 7000 lux ;

- cycle lumière/obscurité : $15 / 9$ heures ;

- températures moyennes : $25 \pm 2^{\circ} \mathrm{C}$ (période d'éclairement) et $18 \pm 2^{\circ} \mathrm{C}$ (période d'obscurité).

La biomasse maximale produite est déduite des mesures quotidiennes de la densité optique des cultures à $750 \mathrm{~nm}$ (spectrophotomètre «PerkinElmer $»)$.

\section{- Analyses physico-chimiques}

Les techniques d'analyses physico-chimiques des lixiviats sont celles recommandées par Afnor (1986). Les références relatives à chaque élément sont les suivantes :

- pH : NFT 90-006; - Turbidité : NFT 90-033; - M.E.S. : NFT 90-105 ; - C.O.T. : T 90-102 ; - N$\mathrm{NO}_{3}$ : NFT 90-012; - N-NH 4 : NFT 90-015; - NKjedahl : NFT 90-110 ; - P-PO 4 et P. totaux : NFT 90-023 ; Hydrocarbures totaux : T 90-114. 


\section{Résultats}

\subsection{Courbes d'étalonnage}

Les figures 1 et 2 représentent respectivement les courbes de croissance de l'algue Selenastrum et les biomasses maximales produites dans les milieux contenant différentes concentrations initiales en P$\mathrm{PO}_{4}$. La cinétique de croissance est marquée par deux phases :

- une phase de croissance exponentielle dont la durée varie de 4 à 10 jours ;

- un plateau ultérieur (phase stationnaire de croissance) en fin de l'expérience ( $21^{\mathrm{e}}$ jour).

La biomasse maximale produite croît linéairement avec la concentration initiale en phosphore du milieu (Fig. 2) selon l'équation :

$\mathrm{BM}=68,91 . \mathrm{N}-3,44 \pm 8,6 \%\left(\mathrm{r}^{2}=0,997\right)$.

BM est la biodisponibilité du phosphore $(\mu \mathrm{g} / \mathrm{l})$, estimée par la biomasse maximale produite.

$\mathrm{N}$ est le nombre de cellules (millions/ml), déterminé par comptage à l'aide de la « cellule de Malassez » (profondeur : $0,200 \mathrm{~mm}$ et surface : $0.0025 \mathrm{~mm}^{2}$ ) et par la densité optique à $750 \mathrm{~nm}$.

\subsection{Réponses des bioessais}

La cinétique de croissance des algues en milieu de culture exempt de phosphore et renfermant 5,10 , 15 ou $20 \%(\mathrm{v} / \mathrm{v})$ de lixiviat (Fig. 3a à 3c), présente des allures qui sont analogues à celles des solutionsétalons (Fig. 1). Ce comportement rejoint les observations de Hanna \& Dauta (1983). La phase de croissance exponentielle n'excède pas 7 jours et le nombre de cellules/ml reste relativement faible $(<106 / \mathrm{ml})$.

La biodisponibilité du phosphore (BM) des lixiviats (Tableau 3) établie à partir de la droite étalon (Fig. 2) montre que les valeurs de ce paramètre sont comprises de 10 à $30 \mu \mathrm{g} / 1$ de $\mathrm{P}-\mathrm{PO}_{4}$. Elle indique par ailleurs des teneurs en phosphore supérieures à celles dosées par voie physico-chimique (les orthophosphates, notamment).

\section{Discussion}

La bioévaluation du potentiel d'eutrophisation des lixiviats de boues de forage pétrolier a abouti aux résultats suivants :

- la cinétique de croissance algale comporte deux phases : une phase de croissance exponentielle dont la durée n'excède pas 7 jours, suivie d'une phase de stabilisation de croissance.

- la biodisponibilité du phosphore estimée par la biomasse maximale produite donne un résultat supérieur à celui obtenu par le dosage physicochimique des orthophosphates.

Dans la cinétique de croissance, la phase exponentielle traduit la facile mobilisation du phosphore des milieux par l'algue Selenastrum. Durant cette phase, le développement de l'organisme-test semble très peu affecté par la présence d'éventuelles

Tableau 3. Biodisponibilité du phosphore (BM) des lixiviats estimée par la biomasse maximale produite.

Table 3. Bioavailability of phosphorus (BM) of leached materials estimating by the yield.

\begin{tabular}{|c|c|c|c|c|c|}
\hline \multirow[t]{2}{*}{ Echantillons } & \multirow[t]{2}{*}{ Paramètres } & \multirow{2}{*}{$\begin{array}{r}\% \text { de lixiviat } \\
5 \%\end{array}$} & \multirow{2}{*}{$\frac{(v / v) \text { ajouté }}{1.0 \%}$} & \multirow{2}{*}{$\begin{array}{c}\text { au milieu de culture } \\
15 \%\end{array}$} & \multirow[b]{2}{*}{$20 \%$} \\
\hline & & & & & \\
\hline \multirow[t]{2}{*}{ Lixiviat 1} & BM $(\mu g / l)$ & 13,5 & 15,3 & 12,9 & 14,4 \\
\hline & \% P.T & 16 & 18,7 & 15 & 17,5 \\
\hline \multirow[t]{2}{*}{ Lixiviat 2} & $B M(\mu g / l)$ & 17,6 & 14,4 & 10,3 & 8,7 \\
\hline & \% P.T & 97,7 & 80 & 57,2 & 48,3 \\
\hline \multirow[t]{2}{*}{ Lixiviat 3} & $B M(\mu g / l)$ & 22,5 & 27,4 & 25 & 17,6 \\
\hline & $\%$ P.T & 83,3 & 100 & 92,6 & 65,1 \\
\hline
\end{tabular}


substances inhibitrices de croissance dans les lixiviats (Bolier \& Donze 1989) ; ce comportement peut être relié à la non (ou très faible) interférence du phosphore avec d'autres éléments des milieux de culture. La croissance nulle traduit un épuisement des milieux en phosphore biodisponible. Par ailleurs, les apports de phosphore libéré des cellules mortes ou des dissociations enzymatiques, paraissent insuffisants pour engendrer des reprises significatives de croissance. Le plateau de croissance nulle peut être aussi consécutif à la complexation du phosphore $\left(\mathrm{PO}_{4}{ }^{3-}\right.$ notamment) avec d'autres éléments comme les produits d'excrétion cellulaire, les ions $\mathrm{Ca}^{2+}$ et/ou métalliques. Il est montré que, dans l'eau,

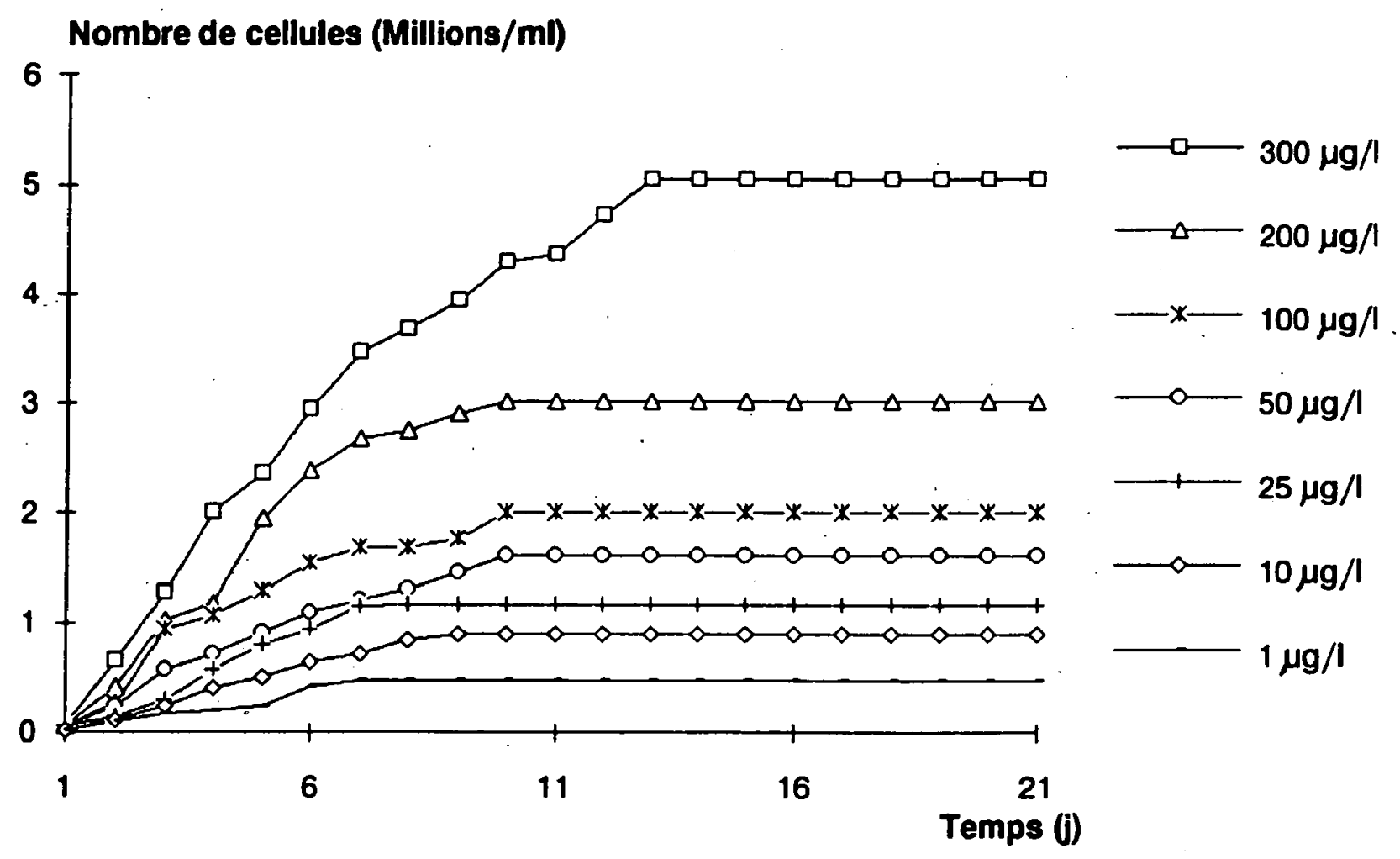

Fig. 1. Courbes de croissance de Selenastrum en fonction de la concentration initiale de $\mathrm{P}-\mathrm{PO}_{4}$.

Fig. 1. Growth curves of Selenastrum in relation to the initial concentration of $\mathrm{P}_{-} \mathrm{PO}_{4}$.

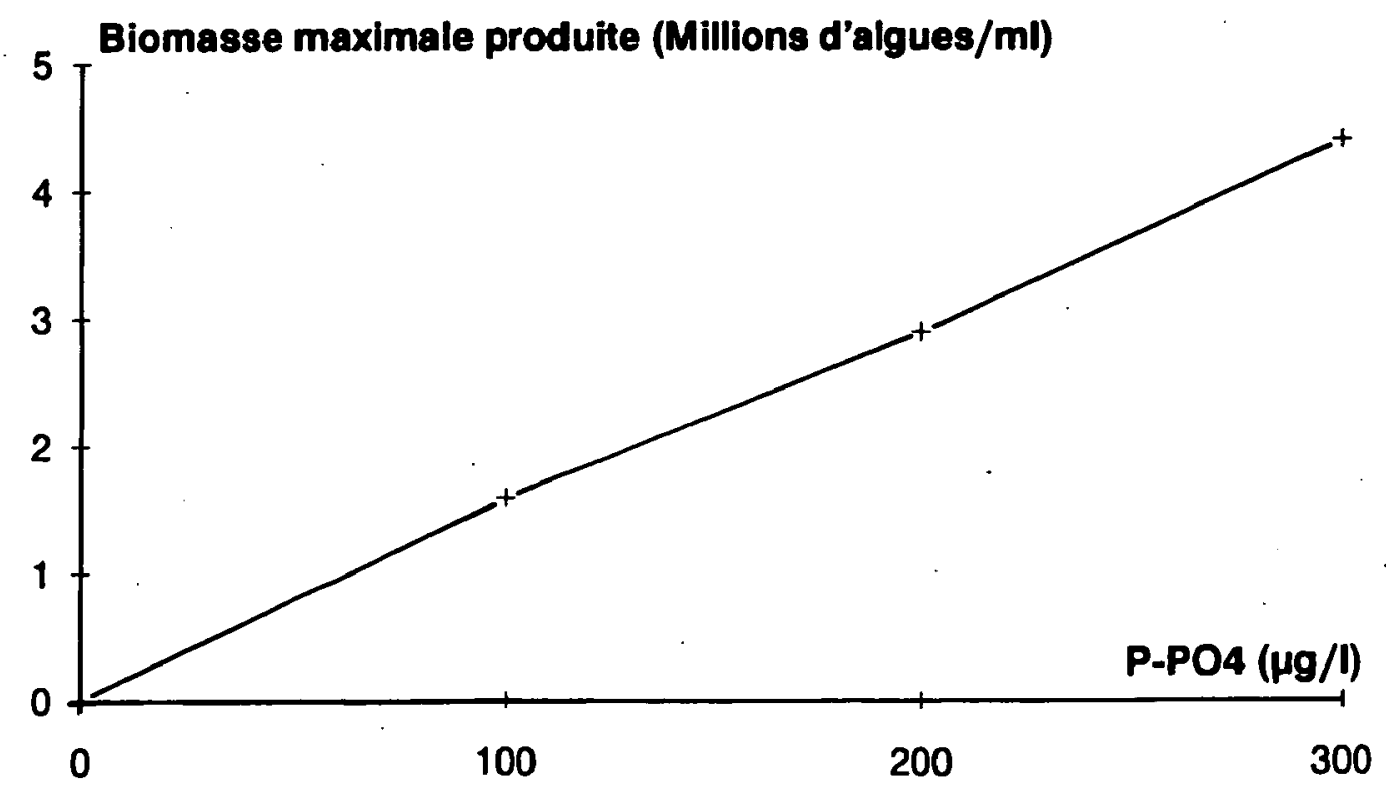

Fig. 2. Biomasse maximale produite en fonction de la concentration initiale de $\mathbf{P}-\mathrm{PO}_{4}$.

Fig. 2 Yield in relation to the initial concentration of $\mathrm{P}-\mathrm{PO}_{4}$. 

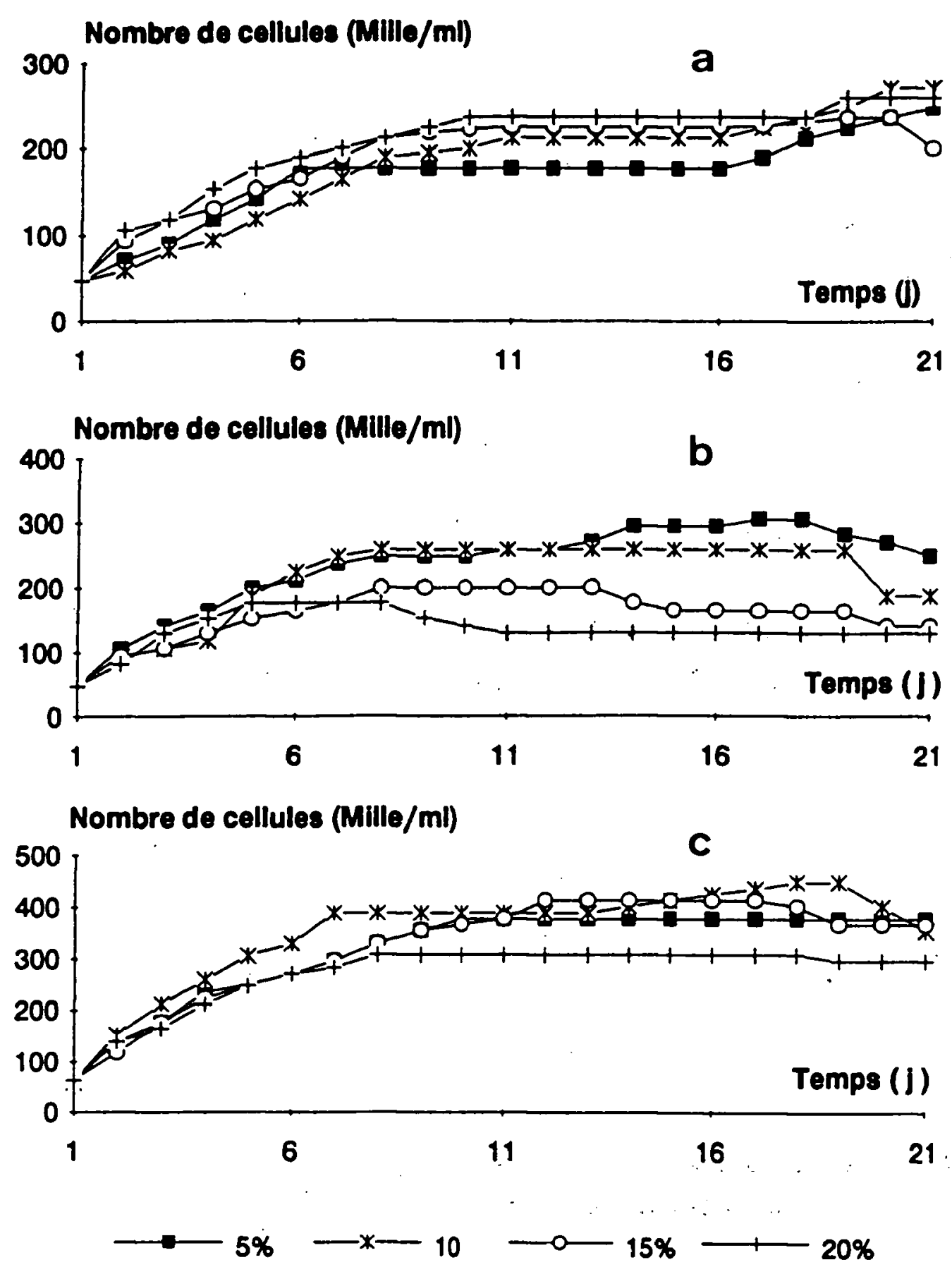

Fig. 3. Courbes de croissance de Selenastrum en milieu de culture exempt de phosphore et renfermant $5,10,15$ ou $20 \%$ (v/v) de lixiviat de boues de forage pétrolier.

Fig. 3. Growth curves of Selenastrum in culture medium without phosphorus and containing 5, 10, 15 or $20 \%$ (v/v) of leached material from petroleum drilling mud.

le $\mathrm{PO}_{4}{ }^{3-}$ s'associe aux éléments métalliques $(\mathrm{Fe})$ pour former des complexes stables (Gatcher et al. 1989). Ces complexes sont très insolubles (Stumm \& Morgan 1981) et leurs cinétiques de diffusion sont très lentes (Van der Zee et al. 1989).

Les teneurs relativement élevées du phosphore estimées par la biomasse maximale produite sont sans doute liées à deux phénomènes majeurs : le prétraitement des lixiviats (autoclavage) et la méthode d'estimation de la biomasse maximale.
L'autoclavage des lixiviats $\left(102^{\circ} \mathrm{C}, 15 \mathrm{mn}\right)$ tend à favoriser l'augmentation de leur potentiel trophique ; ce traitement induit par hydrolyse des hausses de teneur des orthophosphates. De plus, l'autoclavage est susceptible de réduire la toxicité ou la capacité inhibitrice de certaines substances dont il modifie la structure (Hanna 1985). Il est aussi responsable de la destruction des microorganismes dont la lyse libère du phosphore dans les milieux de culture. 
Une autre cause de surévaluation du phosphore biodisponible par rapport au phosphore dosé par voie physico-chimique est la mesure de la biomasse maximale produite déduite des mesures quotidiennes des densités optiques des cultures à $750 \mathrm{~nm}$. De fait, les mesures effectuées à cette longueur d'onde prennent également en compte la densité des cellules algales et des particules en suspension.

\section{Conclusion}

Les résultats obtenus au cours de cette étude montrent que les lixiviats testés sont très faiblement eutrophisants au point de vue phosphore. Ces observations complètent les conclusions de recherches antérieures menées par Elf Aquitaine Production : l'enfouissement technique (qui consiste à enterrer les boues stabilisées par des liants hydrauliques tels que le ciment ou la chaux) et l'épandage des boues non stabilisées (utilisées comme amendement des sols agricoles) sont deux techniques qui ne posent pas de problèmes particuliers vis-à-vis de l'environnement, du moins pour le phosphore. On peut raisonnablement penser que ces deux techniques peuvent être mises en cuvre pour optimiser la stratégie de gestion de ces types de déblais.

\section{Remerciements}

Les auteurs tiennent à remercier la société Elf-Aquitaine Production qui a assuré le financement de ce projet. Leurs remerciements vont également au CEMAGREF de Bordeaux pour la fourniture de la souche d'algue utilisée.

\section{Travaux cités}

AFNOR. 1986. - Eaux. Méthodes d'essai.

AFNOR (NFX 31-210). 1988. - Déchets. Essai de lixiviation. Bayle F. 1980. - Acquisition expérimentale de données concernant la dégradation des algues par les microorganismes. Thèse de Doc. $3^{\mathrm{e}}$ cycle, INSA Toulouse, $76 \mathrm{p}$.

Bolier G. \& Donze M. 1989. - On the accuracy interpretation of the growth curves of planktonic algae. Hydrobiologia, $188 / 189: 175-179$.

Bourrelly P. 1966. - Les algues d'eau douce. Tome I : les algues vertes. Coll. Faune et Flores Actuelles ; Ed. N. Boubée et Cie, Paris, 511 p.

Capblancq J. 1990. - Nutrient dynamics and pelagic food wed interaction in oligotrophic environment : an overview. Hydrobiologia. 207 : 1-14.
Cluis D., Couture P., Beguin R. \& Visser S.A. 1988. - Potential eutrophication in rivers ; relationship between produced and exported loads. Schweiz. Z. Hydrol., 50 (2) : 166-181.

Couture P. 1990. - Approches écotoxicologiques pour évaluer les dangers et les risques environnementaux de polluants aux niveaux des populations et communautés phytoplanctoniques. Thèse de Doct. d'Etat ; Université Paul Sabatier, Toulouse, $209 \mathrm{p}$.

Dauta A. 1982. - Conditions de développement du phytoplancton. Etude comparative du comportement de huit espèces en culture : rôle des nutriments, assimilation et stockage intracellulaire. Annls. Limnol., 18 (3) : 33-40.

Gatcher R., Meyer J.S. \& Mares A. 1988. - Contribution of bacteria to release and fixation of phosphorus in lakes sediments. Limnol. Oceanogr., 33 (6) part 2 : 1542-1558.

Hanna M. \& Dauta A. 1983. - Bioassay : a comparative study of three parameters related to phosphorus bioavailability (yield, growth rate and intercellular concentration of phosphorus). Annls. Limnol., 19 (1) : 59-66.

Hanna M. 1985. - Etude comparée de diverses méthodes d'évaluation de la biodisponibilité du phosphore dans l'eau. Thèse de Doct. $3^{e}$ cycle, Université Paul Sabatier, Toulouse, 159 p.

Klapwijk S.P., Bolier C. \& Van Der J. 1989. - The application of algal growth potential test (AGP) to the canals and lakes of western Netherlands. Hydrobiologia, 188/189 : 189-199.

Lacaze J.C. 1987. - Evaluation du degré de pollution des sédiments marins au moyen d'un test sublétal, l'effet sur l'activité photosynthétique de l'algue Phaodactilum tricornutum mesurée par l'assimilation de Cr. Acad. Sci., Paris, 305 (3) : 515-520.

Lacaze J.C., Chesterikoff A. \& Garbon B. 1989. - Bioévaluation des sédiments de la Seine (région parisienne) par l'emploi d'un bioessai basé sur la croissance à court terme de la micro-algue Selenastrum capricornutum Printz, Rev. Sci. de. l'eau, 2 : 405-427.

Rhee G.Y. 1972. - Competition between an algae and bacterium for phosphate. Limnol. Oceanogr., 17 : 505-514.

Saltman S. \& Heuer B. 1985. - A rapid bioassay for detection of photosynthesis inhibitor in water. Prestic. Sci., 16 : 457-462.

Stumm W. \& Morgan J.J. 1981. - Aquatic chemistry. $2^{\mathrm{e}} \mathrm{ed}$. Wiley interscience, New-York : 780 p.

Van der Zee S., Leus F. \& Louer M. 1989. - Prediction of phosphate transport in small colomns with an approximate sorption kinetics model. Wat. Res., 25 (6) : 1353-1365.

Van Donk E., Mur L.R. \& Ringelbert J. 1989. - A study of phosphate limitation in lake Maarsseveen : phosphate uptake kinetics versus bioassays. Hydrobiologia, 188-189 : 201-209.

Watanabe T., Capblancq J. et Dauta A. 1989. - Utilisation de bioessai « in situ » (substrats artificiels) pour caractériser la qualité des eaux de rivières à l'aide du périphyton. Annls Limnol., 24 (2) : 111-125. 